\section{Reflex Reporting of Estimated Glomerular Filtration Rate (eGFR) with Serum Creatinine: A Win-win Situation for both Patients and Physicians}

\section{Sir,}

The burden of chronic kidney disease (CKD) has extensively risen over the past few decades, emerging as an alarming public health concern with drastic implications on patient-centered outcomes. In Pakistan, owing to the markedly high prevalence of metabolic disorders, diabetes, hypertension and obesity, the cases with CKD have also increased in parallel with a reported prevalence of $12.5 \% .{ }^{1}$ Serumcreatinine $(\mathrm{SCr})$ is the most routinely utilised biomarker in clinical practice for the evaluation of kidney function. However, SCr has various limitations, particularly its sensitivity and extrarenal modulation alongside variation with age, gender and muscle mass. Measurement of glomerular filtration rate (GFR) is the gold standard for assessing kidney function, but it is laboursome, time-consuming and requires sophisticated and costly analytical systems hindering their widespread use. To overcome these challenges, calculation of estimated GFR (eGFR) has been widely adopted globally as an alternative. ${ }^{2}$ To date more than 50 different predicting equations have been developed, mostly SCr or serum cystatin-C based, focused on the population specific use from where they have originated. Most of these equations have been established and validated by the application of various modification factors to the forerunner modification of diet in renal disease (MDRD), Cockcroft-Gault (CG) formula and chronic kidney disease epidemiology collaboration (CKD-EPI) equations.

The National Kidney Disease Education Program (NKDEP) has recommended reporting eGFR reflex with every $\mathrm{SCr}$ test requisition for adults. As the estimate is more easily translatable to a patient's kidney function compared to $\mathrm{SCr}$; and at the same time aids practitioners to identify patients with CKD without adding additional cost and patient inconvenience. According to a survey by the College of American Pathologists (CAP), the reporting of eGFR has consistently followed a rising trend since 2003 by the participating laboratories worldwideas depicted in Figure $1 .{ }^{3}$ However, reporting eGFR with $\mathrm{SCr}$ has not been extensively implemented by clinical laboratories in Pakistan in their reporting formats and in most instances physicians rely on manual calculations. In 2014, CKD-EPI Pakistan equation was proposed i.e. (eGFR [CKD-EPI (PK)] $=0.686 \times$ eGFR [CKD-EPI] to the power 1.059), which was also validated by Ahmed etal, with demonstration of better precision and accuracy compared to the conventional equations. ${ }^{4,5}$ Keeping in view the diagnostic yield and marvels provided by the eGFR at no added finances, in perspective of lower middle-income countries, this is hightime to propagate its incorporation reflexly withSCrlaboratory reports.

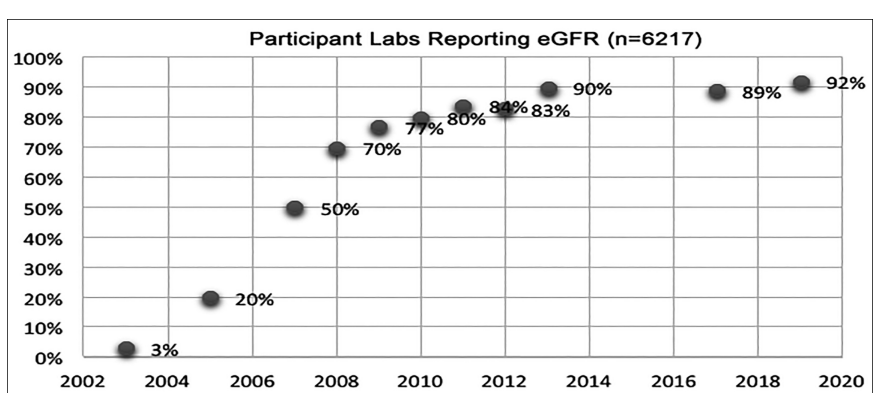

Figure 1: Reporting of eGFR by worldwide clinical laboratories, according to CAP statistics.

\section{CONFLICTOF INTEREST:}

The authors declared no conflict of interest.

\section{AUTHORS' CONTRIBUTION:}

SA: Devised the idea, literature review, and penned the letter.

L): Coordinated letter writing and critical review of the final draft.

\section{REFERENCES}

1. Jessani S, Bux R, Jafar TH. Prevalence, determinants, and management of chronic kidney disease in Karachi, Pakistan - a community based cross-sectional study. BMC Nephrol 2014; 15:90. doi: 10.1186/1471-2369-15-90.

2. Khalid UB, Haroon ZH, Aamir M, Ain QU, Mansoor K, Jaffar SR. Comparison of estimated glomerular filtration rate with both serum creatinine and cystatin C (eGFRcr-cys) versus single analyte (eGFRcr or eGFRcys) using CKD-EPI and MDRD equations in tertiary care hospital settings. J Coll Physicians Surg Pak 2020; 30(7):701-6. doi: 10.29271/ jcpsp.2020.07.701.

3. College of American Pathologists (CAP); kidney biomarkers: The kidney profile order, urine albumin-creatinine ratio (UACR), and estimated glomerular filtration rate (eGFR). Available from: documents.cap.org/documents/2020-a-kidney-biomarkers.pdf (Accessed on 08-06-2021).

4. Jessani S, Levey AS, Bux R, Inker LA, Islam M, Chaturvedi N, et al. Estimation of GFR in South Asians: A study from the general population in Pakistan. Am J Kidney Dis 2014; 63(1):49-58. doi: 10.1053/j.ajkd.2013.07.023.

5. Ahmed S, Jafri L, Khan AH. Evaluation of'CKD-EPI Pakistan'equation for estimated glomerular filtration rate (eGFR): A comparison of eGFR prediction equations in Pakistani population. J Coll Physicians Surg Pak 2017; 27(7): 414.

Sibtain Ahmed and Lena Jafri

Department of Pathology and Laboratory Medicine, The Aga Khan University, Karachi, Pakistan

Correspondence to: Dr. Sibtain Ahmed, Department of Pathology and Laboratory Medicine, The Aga Khan University, Karachi, Pakistan

E-mail: sibtain.ahmed@aku.edu

Received: June 09, 2021; Revised: August 11, 2021;

Accepted: August 20, 2021

DOI: https://doi.org/10.29271/jcpsp.2022.03.411 